\title{
Metabolite and light regulation of metabolism in plants: lessons from the study of a single biochemical pathway
}

\author{
I.C. Oliveira ${ }^{1}$, E. Brenner ${ }^{1}$, \\ J. Chiu', M.-H. Hsieh ${ }^{1}$, \\ A. Kouranovi , H.-M. Lam², \\ M.J. Shin ${ }^{1}$ and \\ G. Coruzzi ${ }^{1}$
}

${ }^{1}$ Department of Biology, New York U niversity, N ew York, NY, USA

${ }^{2}$ Chinese U niversity of Hong Kong, Shatin, New Territories, Hong Kong, China

\section{Correspondence \\ G. Coruzzi \\ Department of Biology \\ New York University \\ Washington Square \\ New York, NY 10003 \\ USA \\ Fax: + 1-212-995-4204 \\ E-mail: Gloria.Coruzzi@ nyu.edu \\ The current address of I.C. O liveira is DuPont Nutrition and Health, \\ Wilmington, DE, USA.}

The current address of M.-H. H sieh is Department of Molecular Biology, Harvard Medical School, Boston, MA, USA.

Research supported by the National Institutes of Health (Grant No. GM 32877), U nited States Department of Energy (Grant N O. DEFG02-92ER20071), and the National Science Foundation (Grant No. MCB 9304913) to G. Coruzzi.

Presented at the XXVIII Annual Meeting of the Brazilian Society of Biochemistry and Molecular Biology, Caxambu, MG, Brazil, May 22-25, 1999.

Received November 16, 2000 Accepted January 3, 2001

\section{Abstract}

We are using molecular, biochemical, and genetic approaches to study the structural and regulatory genes controlling the assimilation of inorganic nitrogen into the amino acids glutamine, glutamate, aspartate and asparagine. These amino acids serve as the principal nitrogentransport amino acids in most crop and higher plants including Arabidopsis thaliana. We have begun to investigate the regulatory mechanisms controlling nitrogen assimilation into these amino acids in plants using molecular and genetic approaches in Arabidopsis. The synthesis of the amide amino acids glutamine and asparagine is subject to tight regulation in response to environmental factors such as light and to metabolic factors such as sucrose and amino acids. For instance, light induces the expression of glutamine synthetase (GLN2) and represses expression of asparagine synthetase (ASN1) genes. This reciprocal regulation of GLN2 and ASN1 genes by light is reflected at the level of transcription and at the level of glutamine and asparagine biosynthesis. Moreover, we have shown that the regulation of these genes is also reciprocally controlled by both organic nitrogen and carbon metabolites. We have recently used a reverse genetic approach to study putative components of such metabolic sensing mechanisms in plants that may be conserved in evolution. These components include an Arabidopsis homolog for a glutamate receptor gene originally found in animal systems and a plant PII gene, which is a homolog of a component of the bacterial Ntr system. Based on our observations on the biology of both structural and regulatory genes of the nitrogen assimilatory pathway, we have developed a model for metabolic control of the genes involved in the nitrogen assimilatory pathway in plants.

\section{Light and metabolic regulation of genes involved in nitrogen metabolism}

In non-legume plants, nitrogen assimilation begins with the uptake of inorganic nitrogen, nitrate or ammonium, from the soil.

\section{Key words}

- Amino acids

- Carbon

- Ammonium

- Nitrogen assimilation

- Gene expression

- Arabidopsis thaliana
Nitrate is subsequently reduced to ammonium $\left(\mathrm{NH}_{4}^{+}\right)$by the sequential action of nitrate reductase and nitrite reductase $(1,2)$. The resulting ammonium is then assimilated into an organic form as glutamate and glutamine. These amino acids are the nitrogen donors in the biosynthesis of essentially all 
Figure 1. Light and metabolites cause a reciprocal effect on Arabidopsis GLN2 and ASN1 gene expression. The mRNA levels of ASN1 are repressed to nearly undetectable levels in lightgrown plants (lane 8) and are strongly enhanced in dark-adapted plants (lane 9). By contrast, the low, undectable levels of GLN2 mRNA from plants grown in the dark (lane 2) are highly elevated in light-grown plants (lane 1). Sucrose can partially mimic the effects of light by causing the induction of GLN2 and repressing the expression of ASN1 mRNA in the dark (lane 10). In the dark, the light-mimicking effects of sucrose can be antagonized by treatment with amino acids (AA) (GLN2, lanes 4 to 7 and ASN1, lanes 11 to 13 ) with each AA affecting the expression of GLN2 and ASN1 to different extents. The differential effects of the AA on both GLN2 and ASN1 mRNA levels may be explained by the possibility that each AA exerts its effects through different but partially overlapping pathways. However, one cannot rule out differences due to rate of uptake or metabolism. PHY, phytochrome; GLN2, Arabidopsis GS2 (glutamate synthetase) gene; ASN1, Arabidopsis gene AS1 (asparagine synthetase) gene. other amino acids and other important nitrogen-containing compounds such as nucleic acids, chlorophyll, hormones and products of secondary metabolism $(2,3)$. The enzymes responsible for the biosynthesis of glutamine and glutamate are glutamine synthetase (GS), glutamate synthase and glutamate dehydrogenase. Following conversion of inorganic nitrogen into glutamine and glutamate, the assimilated nitrogen can be channeled into aspartate and asparagine by the enzymes aspartate aminotransferase and asparagine synthetase (AS), respectively. These enzymes are involved to varying degrees in the three major processes of nitrogen assimilation in plants: a) primary nitrogen assimilation, b) reassimilation of photorespiratory ammonium, and c) reassimilation of "recycled" nitrogen.

Assimilation of inorganic nitrogen and carbon into organic matter has marked effects on different aspects of plant productivity such as biomass and crop yield $(4,5)$. Nitrogen deprivation has been reported to cause a number of metabolic deficiencies in plants including reduced photosynthetic capacity and carboxylation efficiency (6). Therefore, the enzymes of the nitrogen assimilatory pathway play a critical role in many processes vital for plant growth and development. It is, therefore, not surprising that the expression and function of their structural genes are under tight regulation at both transcriptional and post-transcriptional levels. To maintain a correct carbon to nitrogen ratio in the plant, complex mechanisms must have evolved in order to maintain this delicate balance while providing the necessary organic nitrogen supply to the plant. We have begun to understand the nature of the signaling molecules involved in regulating this pathway and to uncover components of putative amino acid/carbon-sensing systems involved in such regulation.

We have shown that environmental cues (e.g., light and nutrient availability) are key factors regulating genes involved in nitrogen assimilation (Figure 1). Light serves to coordinate assimilation of inorganic nitrogen with available carbon backbones produced during photosynthesis. Furthermore, it appears that the biochemical substrates and products of nitrogen assimilation (mainly carbon and amino acids) or their derived metabolites may act as "messengers" mediating the regulation of their biosynthetic genes. Because light and metabolites regulate genes involved in nitrogen assimilation, our studies have
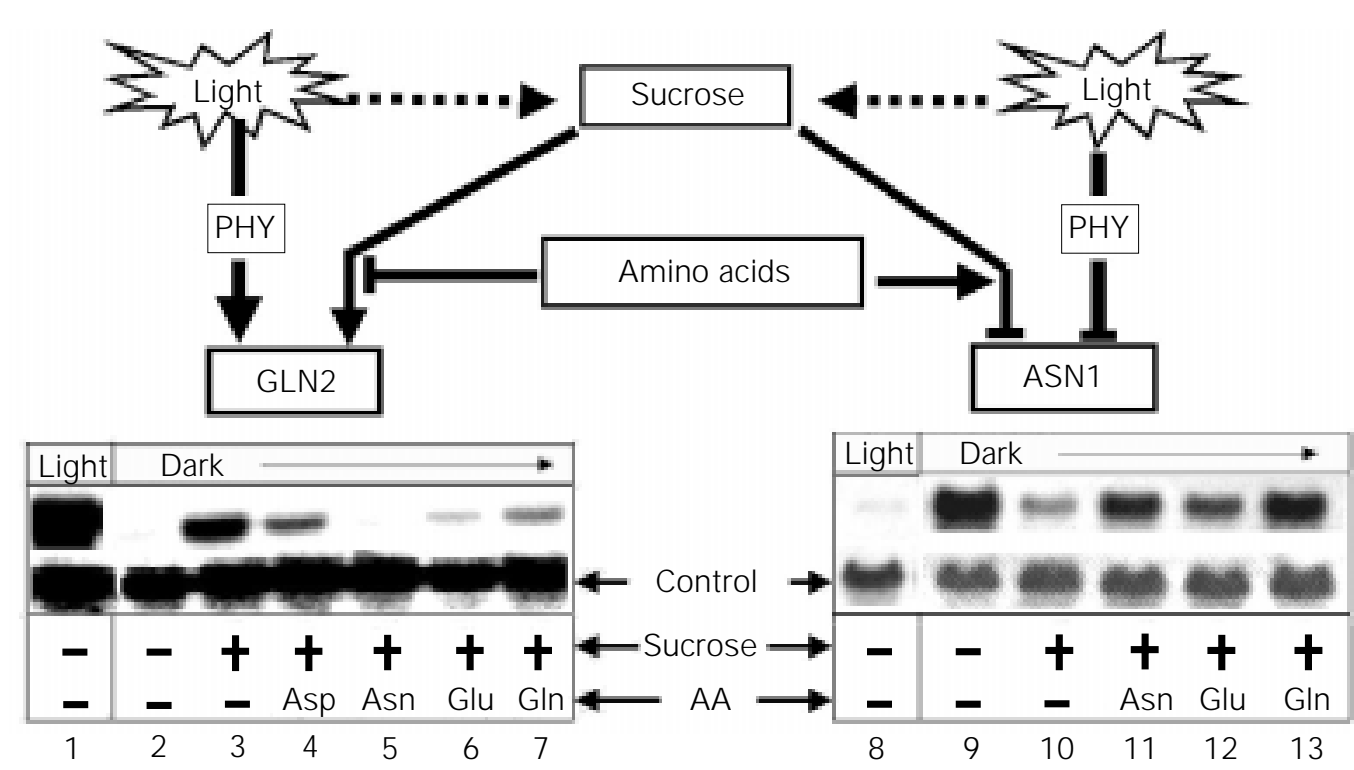
attempted to identify components of such systems, with a special emphasis on mechanisms conserved in biology. Herein, we will highlight the information we have collected in the past several years with regard to defining components involved in light and metabolic regulation of genes involved in nitrogen assimilation using molecular and genetic approaches in Arabidopsis.

\section{Glutamine synthetase and asparagine synthetase: model target genes for the study of light and metabolic sensing}

Asparagine and glutamine are the most abundant free amino acids found in the xylem and phloem, respectively, a fact supporting their role as key nitrogen-transport compounds $(7,8)$. The enzymes responsible for the biosynthesis of asparagine and glutamine are AS and GS, respectively. A particularly interesting point regarding the genes coding for the enzymes AS and GS is that their expression is reciprocally regulated by both light and metabolites $(9,10)$ (see also Figure 1). As such, the pattern of gene expression of AS and GS can serve as a tool for the study of both the positive and negative regulatory effects of light and metabolites in the expression of plant genes coding for enzymes involved in nitrogen metabolism.

The availability of cDNAs and promoters for both GS and AS in different plant species has helped our understanding of their biological roles and gene regulation. To date there are a number of cloned genes for both GS (11-21) and AS (22-31) from a variety of species including Arabidopsis thaliana (3234).

The first insight into the reciprocal regulation of the AS/GS genes came from studies on the effects of light on their expression. For instance, the study of the expression of the nuclear genes encoding the chloroplastic form of GS, GS2, in pea (35), maize (17), Phaseolus (36) and Arabidopsis (34) has revealed that the gene for this GS isoform is tightly regulated by light in a process at least in part mediated by phytochrome $(35,37)$. However, the levels of gene expression for the cytosolic GS1 are not significantly affected by light (37). In contrast to the situation with GS2, light was found to repress accumulation of AS1 mRNA levels in a variety of plants including Arabidopsis, Pisum, Medicago, Glycine, Triphysaria, and Phaseolus (24,26,28-30,32). The negative effect of light on AS gene expression was also shown to be mediated, at least in part, by phytochrome in Arabidopsis and pea (30,32). Further analysis indicated that the repression of AS1 by light involves a calcium/ cGMP-dependent signaling pathway downstream of phytochrome (38). Light regulation of both of AS1 and GS2 can be explained by transcriptional mechanisms since putative cis-acting light regulatory elements, which are required for phytochrome-mediated regulation, have been identified in both the AS1 and GS2 promoters (38-40).

Light can affect plant gene expression by acting directly via phytochrome activation or indirectly via changes in levels of carbon metabolites. One well-described indirect effect of light is the activation of photosynthesis leading to a subsequent increase in the biosynthesis of carbon metabolites. Indeed, light can also modulate GS gene expression in Arabidopsis via an increase in the levels of hexoses and/or non-hexose carbon metabolites. For instance, sucrose treatment can induce the accumulation of mRNA for chloroplastic GLN2 and cytosolic GLN1 in a time-dependent and dose-dependent manner in the absence of light (37). Interestingly, the non-hexose carbon source 2-oxoglutarate can also positively affect the expression of the cytosolic forms of GS. One possible explanation for this finding is that 2-oxoglutarate is particularly important as a carbon level indicator in non-photosynthetic tissues such as roots where the cytosolic GS1 isoforms are most abundantly expressed (41). AS1 
gene expression has also been shown to be subject to regulation by levels of carbon in the form of sugars. Similar to the regulation by light, sucrose too has been shown to repress AS1 mRNA levels in Zea mays, Phaseolus vulgaris, and A. thaliana $(22,28,32)$. In addition to ASN1, two other Arabidopsis AS genes, ASN2 and ASN3, were isolated by functional complementation of a yeast asparagine auxotroph (33). Interestingly, ASN2 is reciprocally regulated with respect to light and carbon metabolites compared to ASN1 genes. ASN2 is induced by light whereas all other known regulated plant AS genes, including ASN1, are repressed by light. It is thought that the differential regulation of ASN2, as compared to ASN1, reflects a difference in the roles these enzymes are thought to play in nitrogen metabolism in vivo (33).

While some progress has been made in understanding sugar sensing and signaling, very little is known about amino acid sensing and signaling in plants. Analysis of free amino acid content by HPLC in A. thaliana, as observed for other species including crop plants, revealed that the four amino acids, glutamine, glutamate, aspartate and asparagine, make up to $64 \%$ of the total free amino acids found in crude leaf extracts $(42,43)$. Amino acids have been reported to be potent signaling molecules in non-plant systems and modulators of enzyme activity and gene expression in plant systems $(32,33$, 37,44-46). In addition, substantial levels of amino acids (mainly asparagine and glutamine) can be detected in phloem and xylem of plants $(8,43,47)$. Taken together, these observations indicate the interesting possibility that amino acids themselves act as "messengers" mediating the regulation of plant gene expression. Indeed, we have observed that different amino acids can antagonize the sucrose induction of individual GS genes to different extents (37). For instance, asparagine was the most effective amino acid downregulating sucrose-induced GS
mRNA accumulation. By contrast, aspartate was the least effective. These differential effects of the amino acids on sucrose-induced GS gene expression can be explained as follows: 1) each amino acid may elicit its effects through different but partially overlapping pathways, 2) each amino acid may be taken up at different rates, and/or 3) different amino acids may be "cleared" from the plant or accumulate in different organs to variable extents. The results obtained for GS regulation by carbon and amino acids in plants are reminiscent of a similar mechanism in Escherichia coli $(48,49)$. The assimilation of inorganic nitrogen into glutamine in $E$. coli is regulated by the relative internal levels of glutamine to 2-oxoglutarate (see below).

Several studies have also begun to explore the effects of organic nitrogen in the form of amino acids on AS regulation. Studies on Arabidopsis have shown that ASN1 mRNA levels were maximally induced in dark-adapted plants in the absence of sucrose (32). The application of exogenous amino acids under these conditions did not affect gene expression (32). However, ASN1 mRNA levels were repressed by sucrose in dark-adapted Arabidopsis plants, and this repression was partially relieved by the addition of the amino acids asparagine, glutamine and glutamate. ASN2 displays a pattern of regulation by amino acids that is reciprocal to that of ASN1. Amino acids (aspartate, asparagine, and glutamine) were found to induce AS mRNA levels in starved corn roots (22). Interestingly, this effect of organic nitrogen was suppressed by the presence of glucose (22). Further investigations should help clarify the specific nature of the signal by which amino acids modulate gene expression and which amino acids or their derivatives are the effectors of this gene regulation in plants.

The complex regulatory mechanisms governing the expression of AS and GS genes by light, carbon and amino acids suggest that 
the regulation of these genes are subject to tight modulatory cues. This complex regulation may provide important clues about the regulation of nitrogen assimilation and transport in plants. Based on current biochemical and molecular knowledge of the regulation of AS and GS genes by light and metabolites, one can draw a model for their roles in nitrogen assimilation. The amino acids asparagine and glutamine are differentially represented in phloem exudates and xylem bleeding sap. Glutamine is more abundant in both whole leaf extracts and phloem exudates of light-grown plants. Asparagine was found at higher levels in dark-adapted $A$. thaliana plants $(42,43)$. In addition, the relative abundance of the amino acids asparagine and glutamine correlates well with the pattern of expression of the genes coding for their biosynthetic enzymes. These observations are in line with the storage and transport functions of asparagine and glutamine: in the presence of light, when carbon skeletons are abundant, ammonia is assimilated via GS into glutamine, which is used in countless anabolic reactions. By contrast, in the dark, when carbon skeletons are limiting, glutamine $(5 \mathrm{C}: 2 \mathrm{~N})$ is converted to asparagine $(4 \mathrm{C}: 2 \mathrm{~N})$, an inert, "carbon-efficient" amino acid used for nitrogen storage by AS.

\section{Integration of light, carbon and nitrogen as signals in plants: conserved mechanisms in nature?}

\section{Nitrogen regulatory system in bacteria: regulation of carbon and nitrogen cross-talk}

Studies of gene expression in higher plants (such as the one described in the previous section) suggest a coordination of nitrogen and carbon metabolism to sustain balanced growth. In prokaryotes, carbon and nitrogen metabolites function as intracellular signals indicating nitrogen and carbon sufficiency. The discovery of the plant gene GLB1 encoding a plant homolog of the PII gene found in enteric bacteria, cyanobacteria and archaea is the first indication that plants may contain a metabolite-sensing system similar to that of prokaryotes (50). The PII protein identified in enteric bacteria is a component of the nitrogen regulatory system that, in addition to PII, includes three bifunctional enzymes, uridylyltransferase/uridylyl-removing enzyme (UTase/UR), adenylyltransferase/ adenylyl-removing enzyme (ATase/AR), and histidine protein kinase/phosphatase (NRII), in addition to the enhancer-binding protein NRI (51). In enteric bacteria PII functions as a transmitter of metabolic signals that in turn regulate the enzymatic activity of GS and the expression of the nitrogen-regulated operon. The nitrogen-regulated operon includes GS and a number of other genes involved in nitrogen metabolism (52).

Glutamine and 2-oxoglutarate are thought to work as metabolic signals in bacterial cells to indicate the relative levels of nitrogen and carbon, respectively (53). High glutamine/2-oxoglutarate ratios lead to the repression of ammonium assimilation into glutamine. In contrast, low glutamine/2-oxoglutarate ratios lead to the activation of ammonium assimilation into glutamine (54). Mechanistically, the levels of glutamine in the cytoplasm are "sensed" by UTase, which uridylylates or removes a UMP residue from PII depending on glutamine concentration.

To date, PII proteins have been identified in three species of higher plants, i.e., Arabidopsis, Castor bean and soybean. Primary structure analysis indicates that all plant PIIs share about $50 \%$ identity with their bacterial homologs. It is noteworthy that highest identity is localized in two PII signature motifs that coincide with functionally important regions representing surface exposed loops (T-loop and B-loop). Structural and mutagenesis studies of bacterial PII suggest that the T-loop is involved in interaction with the receptor molecules UTase/UR, ATase/AR and NRII $(50,54)$. The B-loop is presumably important for interaction with the small-mol- 
ecule effectors such as 2-oxyglutarate and ATP. The structural identity of plant and bacterial PIIs suggests that these proteins may perform similar functions.

In contrast to proteobacteria, which contain two genes encoding PII proteins (GlnB and GlnK), there appears to be one PII gene in higher plants (50). Protein primary structure analysis and cellular organelle localization studies suggest that PII from Arabidopsis is a nuclear-encoded chloroplast-localized protein (50). The chloroplastic localization of Arabidopsis PII suggests that this protein may represent an ancestral mechanism of metabolite sensing common to bacteria and plants. Expression of the PII gene in plants is positively regulated by light and sucrose and negatively regulated by amino acids (50). This regulation is similar to that of other nitrogen-assimilatory genes such as GS and nitrate reductase. The indication that PII protein may be involved in the regulation of nitrogen metabolism in plants came from experiments with transgenic Arabidopsis plants, which overexpress the PII protein (50). In these experiments PII-overexpressing plants were subject to a bioassay to determine whether they had defects in C:N responses. When Arabidopsis plants were grown on media containing a high concentration of sucrose without any nitrogen source, the seedlings accumulated high levels of anthocyanin. The sucrose-induced anthocyanin accumulation in these Arabidopsis seedlings could be relieved by the addition of inorganic nitrogen (ammonium or nitrate) or organic nitrogen in the form of glutamine. The experiments showed that the PII overexpressors accumulated a significantly higher amount of anthocyanin when grown on medium in the presence of $3 \%$ sucrose and $1 \mathrm{mM}$ glutamine as the only source of nitrogen, compared to wild-type (50).

The functional and mechanistic role of PII protein in nitrogen metabolism in higher plants remains to be investigated. However, based on the present experimental data we can hypothesize that PII may be a metabolic regulator involved in sensing of cellular amino acid or carbon status and in the coordination of carbon and nitrogen metabolism in plants.

\section{Animal systems and glutamate receptors: light and metabolites cross-talk}

One of the more interesting signaling systems found in biology exists in the nervous system of animals where certain amino acids act as neurotransmitters. The most common amino acid neurotransmitter in the brain is glutamate and, accordingly, glutamate receptors (GluRs) have been implicated in a range of neurological activities such as memory, vision and a variety of other functions in the brain (44). One large class of GluRs is the one composed of ionotropic GluRs (iGluRs). These ligand-gated ion channels are critical since they function to relay rapid synaptic transmission $(55,56)$. iGluRs can be subdivided into different classes according to their relative sensitivities to different chemical agonists (e.g., AMPA, KA, NMDA) and each class in turn is composed of a large gene family (44).

To determine whether plants may sense amino acids via glutamate receptors, $A$. thaliana $\mathrm{EST}$ sequences were analyzed for genes with high sequence similarity to iGluRs. We have characterized four members of the GluR gene family (AtGLR1 to 4) in A. thaliana $(57,58)$. According to primary sequence analysis, Arabidopsis AtGLR and animal iGluRs share extensive sequence identity in important functional domains defined in animal iGluRs. These include the two ligand-binding domains (GlnH1 and 2), the three transmembrane regions (M1 to 3), and the pore (P) (which was previously designated as transmembrane region 2). In particular, the highest similarity between $A t G L R \mathrm{~s}$ and animal iGluRs is observed in M2 (previously known as M3) in which the amino acid per- 
cent identity is above $60 \%$. In addition to primary sequence similarity, the secondary structure of AtGLRs, i.e., the membrane topology, is also analogous to animal iGluRs based on hydropathy plots and transmembrane prediction programs (58).

We performed a phylogenetic analysis of the iGluR family of plants and animals (57). The results of our parsimony and neighborjoining analyses indicated that AtGLRs diverged from animal iGluRs before the divergence of the various animal iGluR subtypes (AMPA, KA, NMDA, and delta). This suggests that GluRs represent a primitive signaling mechanism that existed before plants and animals diverged. Moreover, our phylogenetic analysis established the relationship among members of the AtGLR gene family. According to our analysis, AtGLR2 and AtGLR4 are more closely related to each other than to either AtGLR1 or AtGLR3.

As a first attempt to investigate the in vivo function(s) of AtGLR gene products in plants, we treated Arabidopsis seedlings with the iGluR antagonist DNQX, which is known to block KA/AMPA iGluRs in animals (58). DNQX treatment of Arabidopsis plants causes hypocotyl elongation and reduction in chlorophyll levels. This effect is specific for light-grown Arabidopsis in that plants grown in the dark on DNQX have normal length hypocotyls. DNQX-induced hypocotyl shortening is reminiscent of the " $h y$ " mutations in Arabidopsis, which are impaired in photomorphogenic light signal transduction. This suggests that $A t G L R$ s may be involved in light signal transduction.

\section{Conclusion and future prospects}

We have discussed the power of using the combined approaches of molecular biol- ogy and genetics to study the environmental and metabolic cues underlying the control of nitrogen assimilation in plants. The demonstration that genes coding for enzymes in this pathway can have their expression modulated by metabolic cues opened a whole new window on both metabolic signal sensing and transduction in plants. Using the current vast array of molecular-genetic tools available for the model plant Arabidopsis, it is now possible to thoroughly delineate the mechanisms responsible for the regulation of this pathway, as well as to characterize the signal-transducing elements involved. Our studies have attempted to identify components of such amino acid and/or carbonsensing systems, with special emphasis on mechanisms conserved in biology. The results of these studies support the notion that, in order to maintain a correct carbon to nitrogen ratio in the plant and still provide the necessary organic nitrogen supply to the plant, complex mechanisms have evolved. We have begun to understand the nature of the signaling molecules involved in regulating this pathway and to uncover components of putative amino acid/carbon-sensing systems involved in such regulation. How many different signals are involved, how they reach their "sensors" in the plant and where these "sensors" are located, what the actual mediators of metabolic sensing are and what the molecules involved in this signal transduction pathway are: these are a few of the questions to be answered in the coming years.

\section{Acknowledgments}

The authors thank Joan Odell and William Hitz for critical reading of the manuscript. 


\section{References}

1. Crawford NM (1995). Nitrate: Nutrient and signal for plant growth. Plant Cell, 7: 859868.

2. Lea PJ (1993). Nitrogen metabolism. In: Lea PJ \& Leegood RC (Editors), Plant Biochemistry and Molecular Biology. John Wiley \& Sons, New York, 155-180.

3. Rose AB \& Last RL (1994). Molecular genetics of amino acid, nucleotide, and vitamin biosynthesis. In: Meyerowitz EM \& Sommerville CR (Editors), Arabidopsis. Cold Spring Harbor Laboratory Press, Plainview, 835-879.

4. Lawlor DW, Kontturi $M \&$ Young AT (1989). Photosynthesis by flag leaves of wheat in relation to protein, ribulose bisphosphate carboxylase activity and nitrogen supply. J ournal of Experimental Botany, 40: 43-52.

5. Mattsson $M$, J ohansson $E$, Lundborg $T$, Larsson M \& Larsson CM (1991). Nitrogen utilization in N-limited barley during vegetative and generative growth. I. Growth and nitrate uptake kinetics in vegetative cultures grown at different relative addition rates of nitrate-N. J ournal of Experimental Botany, 42: 197-205.

6. Delgado E, Mitchell RAC, Parry MA, Driscoll SP, Mitchell VJ \& Lawlor DW (1994). Interacting effects of $\mathrm{CO}_{2}$ concentration, temperature and nitrogen supply on the photosynthesis and composition of winter wheat leaves. Plant, Cell and Environment, 17: 1205-1213.

7. Lea PJ \& Miflin BJ (1980). Transport and metabolism of asparagine and other nitrogen compounds within the plant. In: Miflin BJ (Editor), The Biochemistry of Plants. Vol. 5. Amino Acids and Derivatives. Academic Press, New York, 569-607.

8. Urquhart AA \& J oy KW (1981). Use of phloem exudate technique in the study of amino acid transport in pea plants. Plant Physiology, 68: 750-754.

9. McGrath RB \& Coruzzi GM (1991). A gene network controlling glutamine and asparagine biosynthesis in plants. Plant J ournal, 1: 275-280.

10. Ngai N \& Coruzzi G (1998). Dissecting light repression of asparagine synthetase gene in Arabidopsis. In: LoSchiavo F, Raikhel N, Last R \& Morelli G (Editors), Cellular Integration of Signaling Pathways in Plant Development. Springer Verlag, Amsterdam, 147-157.

11. Cullimore J V, Gebhardt C, Saarelainen R, Miflin BJ , Idler KB \& Barker RF (1984). Glutamine synthetase of Phaseolus vulgaris L.: organ-specific expression of a multigene family. Journal of Molecular and Applied Genetics, 2: 589-599.

12. Gebhardt C, Oliver J E, Forde BG, Saarelainen R \& Miflin BJ (1986). Primary structure and differential expression of glutamine synthetase genes in nodules, roots and leaves of Phaseolus vulgaris. EMBO J ournal, 5: 1429-1435.

13. Hirel B, Bouet C, King B, Layzell B, J acobs F \& Verma DPS (1987). Glutamine synthetase genes are regulated by ammonia provided externally or by symbiotic nitrogen fixation. EMBO J ournal, 6: 11671171.

14. Li M-G, Villemur R, Hussey PJ, Silflow CD, Gantt J S \& Snustad DP (1993). Differential expression of six glutamine synthetase genes in Zea mays. Plant Molecular Biology, 23: 401-407.

15. Lightfoot DA, Green NK \& Cullimore JV (1988). The chloroplast located glutamine synthetase of Phaseolus vulgaris L.: Nucleotide sequence, expression in different organs and uptake into isolated chloroplasts. Plant Molecular Biology, 11: 191-202.

16. Roche D, Temple SJ \& Sengupta-Gopalan C (1993). Two classes of differentially regulated glutamine synthetase genes are expressed in the soybean nodule: A nodule-specific and a constitutively expressed class. Plant Molecular Biology, 22: 971983.

17. Sakakibara $\mathrm{H}$, Kawabata $\mathrm{S}$, Takahashi $\mathrm{H}$, Hase T\& Sugiyama T (1992). Molecular cloning of the family of glutamine synthetase genes from maize: Expression of genes for glutamine synthetase and ferrodoxin-dependent glutamate synthase in photosynthetic and non-photosynthetic tissues. Plant and Cell Physiology, 33: 4958.

18. Sakamoto A, Ogawa M, Masumura $T$, Shibata D, Takeba G, Tanaka K \& Fujii S (1989). Three cDNA sequences coding for glutamine synthetase polypeptides in Oryza sativa L. Plant Molecular Biology, 13: 611-614.

19. Snustad PD, Hunsperger J P, Chereskin BM \& Messing J (1988). Maize glutamine synthetase CDNAs: Isolation by direct genetic selection in Escherichia coli. Genetics, 120: 1111-1124.

20. Tingey SV, Tsai F-Y, Edwards J W, Walker EL \& Coruzzi GM (1988). Chloroplast and cytosolic glutamine synthetase are encoded by homologous nuclear genes which are differentially expressed in vivo. J ournal of Biological Chemistry, 263:
9651-9657.

21. Walker EL \& Coruzzi GM (1989). Developmentally regulated expression of the gene family for cytosolic glutamine synthetase in Pisum sativum. Plant Physiology, 91: 702-708.

22. Chevalier C, Bourgeois $E$, J ust $D \&$ Raymond P (1996). Metabolic regulation of asparagine synthetase gene expression in maize root tips. Plant J ournal, 9: 1-11.

23. Davis KM \& King GA (1993). Isolation and characterization of a cDNA clone for a harvest induced asparagine synthetase from Asparagus officinalis L. Plant Physiology, 102: 1337-1340.

24. Delavault $\mathrm{P}$, Estabrook $\mathrm{E}$, Albrecht $\mathrm{H}$ Wrobel R \& Yoder JI (1998). Host-root exudates increase gene expression of asparagine synthetase in the roots of a hemiparasitic plant Triphysaria versicolor (Scrophulariaceae). Gene, 222: 155-162.

25. Downs CG, Pogson BJ, Davies KM \& Amira EC (1995). An asparagine synthetase cDNA clone (Genbank x84448) from broccoli (PCR95-016). Plant Physiology, 108: 1342.

26. Hughes CA, Beard HS \& Matthews BF (1997). Molecular cloning and expression of two cDNAs encoding asparagine synthetase in soybean. Plant Molecular Biology, 33: 301-311.

27. Kuster $\mathrm{H}$, Albus $\mathrm{U}$, Fruhling $\mathrm{M}$, Tchetkova SA, Tikhonovitch IA, Puhler A \& Perlick AM (1997). The asparagine synthetase gene vfas 1 is strongly expressed in the nitrogen-fixing zone of broad bean (Vicia faba L.) root nodules. Plant Science, 124: 89-95.

28. Osuna D, Galvez G, Pineda M \& Aguilar M (1999). RT-PCR cloning, characterization and mRNA expression analysis of a cDNA encoding a type II asparagine synthetase in common bean. Biochimica et Biophysica Acta, 1445: 75-85.

29. Shi L, Twary S, Yoshioka H, Gregerson R, Miller S, Samac D, Gantt J , Unkefer P \& Vance C (1997). Nitrogen assimilation in alfalfa: Isolation and characterization of an asparagine synthetase gene showing enhanced expression in root nodules and dark-adapted leaves. Plant Cell, 9: 13391356.

30. Tsai F-Y \& Coruzzi GM (1990). Dark-induced and organ-specific expression of two asparagine synthetase genes in $\mathrm{Pi}$ sum sativum. EMBO J ournal, 9: 323-332.

31. Waterhouse RN, Smyth A, Massonneau A, Prosser IM \& Clarkson DT (1996). Molecular cloning and characterization of as- 
paragine synthetase from Lotus japonicus: Dynamics of asparagine synthesis in $\mathrm{N}$-sufficient conditions. Plant Molecular Biology, 30: 883-897.

32. Lam H-M, Peng SS-Y \& Coruzzi GM (1994). Metabolic regulation of the gene encoding glutamine-dependent asparagine synthetase in Arabidopsis thaliana. Plant Physiology, 106: 1347-1357.

33. Lam HM , Hsieh MH \& Coruzzi GM (1998). Reciprocal regulation of distinct asparagine synthetase genes by light and metabolites in Arabidopsis thaliana. Plant J ournal, 16: 345-353.

34. Peterman TK \& Goodman HM (1991). The glutamine synthetase gene family of Arabidopsis thaliana: Light-regulation and differential expression in leaves, roots and seeds. Molecular and General Genetics, 230: 145-154.

35. Edwards J W \& Coruzzi GM (1989). Photorespiration and light act in concert to regulate the expression of the nuclear gene for chloroplast glutamine synthetase. Plant Cell, 1: 241-248.

36. Cock J M, Brock IW, Watson AT, Swarup R, Morby AP \& Cullimore J V (1991). Regulation of glutamine synthetase genes in leaves of Phaseolus vulgaris. Plant Molecular Biology, 17: 761-771.

37. Oliveira IC \& Coruzzi G (1999). Reciprocal regulation by carbon and nitrogen metabolites of genes for glutamine synthetase in Arabidopsis. Plant Physiology, 121: 301309.

38. Neuhaus G, Bowler C, Hiratsuka K, Yamagata H \& Chua NH (1997). Phytochrome-regulated repression of gene expression requires calcium and CGMP. EMBO J ournal, 16: 2554-2564.

39. Ngai N, Tsai FY \& Coruzzi G (1997). Lightinduced transcriptional repression of the pea AS1 gene: Identification of cis-elements and trans-factors. PlantJ oumal, 12: 1021-1034.

40. Tjaden G, Edwards JW \& Coruzzi GM (1995). Cis elements and trans-acting factors affecting regulation of a non-photosynthetic light-regulated gene for chloroplast glutamine synthetase. Plant Physiology, 108: 1109-1117.
41. Oaks A (1992). A re-evaluation of nitrogen assimilation in roots. BioScience, 42: 103111.

42. Lam H-M, Coschigano K, Schultz C, MeloOliveira R, Tjaden G, Oliveira I, Ngai N, Hsieh M-H \& Coruzzi GM (1995). Use of Arabidopsis mutants and genes to study amide amino acid biosynthesis. Plant Cell, 7: 887-898

43. Schultz CJ (1994). A molecular and genetic dissection of the aspartate aminotransferase isoenzymes of Arabidopsis thaliana. Doctoral thesis, New York University, New York, NY.

44. Hollmann M \& Heinemann S (1994). Cloned glutamate receptors. Annual Review of Neuroscience, 17: 31-108.

45. Ratajczak L, Ratajczak W \& Mazurowa H (1981). The effect of different carbon and nitrogen sources on the activity of glutamine synthetase and glutamate dehydrogenase in lupine embryonic axes. Physiologia Plantarum, 51: 277-280.

46. Vincentz M, Moureaux T, Leydecker M-T, Vaucheret H \& Caboche M (1993). Regulation of nitrate and nitrite reductase expression in Nicotiana plumbaginifolia leaves by nitrogen and carbon metabolites. Plant J ournal, 3: 315-324.

47. Urquhart AA \& J oy KW (1982). Transport, metabolism, and redistribution of xylemborne amino acids in developing pea shoots. Plant Physiology, 69: 1226-1232.

48. Magasanik B \& Neidhardt FC (1987). Regulation of carbon and nitrogen utilization. In: Neidhardt FC, Ingraham J L, Low $K B$, Magasanik B, Schaechter $M$ \& Umbarger HE (Editors), Escherichia coli and Salmonella typhimurium: Cellular and Molecular Biology. American Society for Microbiology, Washington, DC, 13181325.

49. Neidhardt FC (1987). Multigene systems and regulons. In: Neidhardt FC, Ingraham J L, Low KB, Magasanik B, Schaechter M \& Umbarger HE (Editors), Escherichia coli and Salmonella typhimurium: Cellular and Molecular Biology. American Society for Microbiology, Washington, DC, 13131317.

50. Hsieh MH, Lam HM, Van De Loo F \&
Coruzzi G (1998). A PII-like protein in Arabidopsis: Putative role in nitrogen sensing. Proceedings of the National Academy of Sciences, USA, 95: 13965-13970.

51. J iang P, Peliska J A \& Ninfa AJ (1998). Enzymological characterization of the signal-transducing uridylyltransferase/uridylyl-removing enzyme (EC 2.7.7.59) of Escherichia coli and its interaction with the PII protein. Biochemistry, 37: 1278212794.

52. Shapiro B (1969). The glutamine synthetase deadenylylating enzyme system from E. coli. Resolution into two components, specific nucleotide stimulation, and cofactor requirements. Biochemistry, 8: 659-670.

53. J iang P, Peliska J A \& Ninfa AJ (1998). Reconstitution of the signal-transduction bicyclic cascade responsible for the regulation of Ntr gene transcription in Escherichia coli. Biochemistry, 37: 1279512801.

54. J iang P, Zucker P, Atkinson MR, Kamberov ES, Tirasophon W, Chandran $P$, Schefke BR \& Ninfa AJ (1997). Structure/ function analysis of the PII signal transduction protein of Escherichia coli: Genetic separation of interactions with protein receptors. J ournal of Bacteriology, 179: 4342-4353.

55. Tsien J Z, Huerta PT \& Tonegawa S (1996). The essential role of hippocampal CA1 NMDA receptor-dependent synaptic plasticity in spatial memory. Cell, 87: 13271338.

56. Wisden W \& Seeburg PH (1993). Mammalian ionotropic glutamate receptors. Current Opinion in Neurobiology, 3: 291298.

57. Chiu J, Desalle R, Lam HM, Meisel L \& Coruzzi G (1999). Molecular evolution of glutamate receptors: A primitive signaling mechanism that existed before plants and animals diverged. Molecular Biology and Evolution, 16: 826-838.

58. Lam HM, Chiu J, Hsieh M, Meisel L, Oliveira IC, Shin M \& Coruzzi G (1998). Glutamate receptor genes in plants. $\mathrm{Na}$ ture, 396: 125-126. 